\title{
Progress in Clinical Neurosciences: Sepsis-Associated Encephalopathy: Evolving Concepts
}

\author{
John X. Wilson and G. Bryan Young
}

\begin{abstract}
Systemic sepsis commonly produces brain dysfunction, sepsis-associated encephalopathy, which can vary from a transient, reversible encephalopathy to irreversible brain damage. The encephalopathy in the acute phase clinically resembles many metabolic encephalopathies: a diffuse disturbance in cerebral function with sparing of the brain stem. The severity of the encephalopathy, as reflected in progressive EEG abnormalities, often precedes then parallels dysfunction in other organs. Recent research has revealed a number of potentially important, non-mutually exclusive, mechanisms that have therapeutic implications.
\end{abstract}

\begin{abstract}
RÉSUMÉ: Encéphalopathie associée à la septicémie: concepts en évolution. La septicémie induit communément une dysfonction cérébrale, l'encéphalopathie associée à la septicémie, dont la sévérité varie d'une encéphalopathie transitoire réversible au dommage cérébral irréversible. En phase aiguë, l'encéphalopathie ressemble cliniquement à plusieurs autres encéphalopathies métaboliques: une perturbation diffuse de la fonction cérébrale épargnant le tronc cérébral. L'encéphalopathie, comme en témoigne les anomalies progressives observées à l'ÉEG, précède souvent la dysfonction des autres organes puis évolue parallèlement à cette dysfonction. Des recherches récentes ont révélé plusieurs mécanismes potentiellement importants, qui ne sont pas mutuellement exclusifs et qui ont des implications thérapeutiques.
\end{abstract}

Can. J. Neurol. Sci. 2003; 30: 98-105

Sepsis is the systemic response to microorganisms or their toxins. ${ }^{1}$ There is abundant evidence that this response is produced by inflammatory mediators or cytokines. ${ }^{1}$ Confusion regarding the role of infection has arisen because trauma, burns and pancreatitis may produce the same systemic response with release of inflammatory mediators, in the absence of infection. Thus, the systemic inflammatory response syndrome may or may not have an underlying infectious agent and consists of fever or hypothermia, tachycardia, tachypnea, hyperdynamic circulation, hypercatabolic state and evidence of organ hypoperfusion or organ dysfunction. ${ }^{1}$

Brain dysfunction or encephalopathy is a common accompaniment to systemic inflammatory response syndrome. We have used the term sepsis-associated encephalopathy (SAE) ${ }^{2}$ to define a diffuse or multifocal cerebral dysfunction as a component of infection (i.e., there should be an identified microorganism) with systemic response but without clinical or laboratory evidence of direct brain infection (i.e., we exclude meningitis or macroscopic cerebritis or brain abscesses). We suggest that SAE is preferable to the loosely used term "septic encephalopathy" which to some implies a consistent, direct infection of the central nervous system.

Sepsis-associated encephalopathy is a common problem with serious consequence. In our studies of hospital patients with bacteremia, $87 \%$ had abnormal electroencephalograms (EEG) and $70 \%$ were diagnosed with neurologic symptoms ranging from lethargy to coma. ${ }^{2-4}$ Forty-six percent of these patients with brain dysfunction were in the intensive care unit, with the rest on the wards. Survivors of severe sepsis were often left with debilitating cognitive and behavioral problems that persisted for years or were irreversible. 5

\section{Pathology and pathophysiology}

In fatal cases, watershed brain infarctions from sustained hypotension are rarely encountered, however the brain may show microscopic abnormalities. ${ }^{6}$ Eight of 12 of our patients with postmortem examinations had disseminated microabscesses. The cerebral cortex is the site most commonly involved, but deep structures and even the spinal cord may be affected. Other less common lesions include multiple microscopic infarctions, brain purpura and central pontine myelinolysis. ${ }^{6}$ Some cases may show no abnormality on gross or light microscopic examination.

From the Departments of Physiology (JXW) and Clinical Neurological Sciences (GBY), the University of Western Ontario, London, Ontario, Canada.

RECEIVED APRIL 22. ACCEPTED IN FINAL FORM SEPTEMBER 4, 2002.

Reprint requests to: G.B. Young, Division of Neurology, Sunnybrook and Women's

Health Sciences Centre, 2075 Bayview Avenue, Toronto, ON, Canada M4N 3M5 
The mechanisms of SAE are uncertain. In advanced cases it is likely multifactorial, given the variety of pathological findings. The early, fully reversible cases are not likely associated with structural change and are probably metabolic in nature. Other mechanisms, in addition to the metabolic disturbances, operate in more advanced cases. The following mechanisms have been proposed:

\section{Microvascular disorder}

Reduced cerebral blood flow and increased cerebrovascular resistance are found in human cases of SAE. ${ }^{7,8}$ Microthromboses and microinfarctions may also occur. At least some of these phenomena likely relate to the production of cytokines [e.g., tumor necrosis factor- $\alpha$ (TNF- $\alpha$ ), interferon- $\gamma$ (IFN $\gamma$ ) and interleukins (IL)] and their effects on the production of nitric oxide (NO) by endothelial nitric oxide synthase (eNOS). Inhibition of eNOS leads to impairment of the microcirculation of the brain and other organs by causing vasoconstriction. ${ }^{9}$ In addition, sepsis is a procoagulant state that rests with endothelial dysfunction: endothelial cells convert protein $\mathrm{C}$ to activated protein $\mathrm{C}$ that has anti-inflammatory and profibrinolytic properties. ${ }^{10}$ Indeed, organ dysfunction in sepsis can be improved by the administration of activated protein C. ${ }^{11}$ The brain may be similar to other organs in this respect. The bloodbrain barrier, which normally maintains a homeostatic environment for brain cells, becomes leaky in a dynamic and patchy manner within the first few hours of endotoxemia in experimental animals. ${ }^{12}$ In addition, the differential flux of chemicals across the blood-brain barrier is altered in sepsis; e.g., aromatic amino acids are more readily transported than branched chain amino acids from blood to brain. Inflammatory cytokines and free radicals likely produce these effects on capillary permeability.

\section{Amino acids and neurotransmitter imbalance}

Septic humans and animal models of sepsis both show an increased ratio of aromatic to branched-chain amino acids in the plasma. ${ }^{13-16}$ This altered ratio correlates with decreased norepinephrine, dopamine and serotonin concentrations in the brains of septic rats. There are a number of factors that are yet to be explored, such as the concentration of gamma-amino butyric acid (GABA), the activity of GABA receptors in the brain, and the levels of benzodiazepine-like substances (with inhibitory or sedating properties) and quinolinic acid that are elevated in hepatic dysfunction. ${ }^{17-19}$

\section{Brain microabscesess}

These are of uncertain significance with respect to the pathogenesis of encephalopathy. ${ }^{6}$ Because they are microscopic, they are not detected in life, even with high-resolution MRI scans. There is an association of brain microabscesses with retinal microabscesses, which are a rarity in our experience. ${ }^{1}$ There is no good way of knowing when microabscesses occur in the course of sepsis but, based on the surrounding reaction in the brain, probably they are not all agonal. Indeed, a recent paper found microabscesses to be one of the pathological conditions associated with central autonomic dysfunction in sepsis. ${ }^{20}$ Microabscesses could cause further neuronal damage and dysfunction due to local release of cytokines and other inflammatory mediators.

\section{Failure of other organs}

Hepatic or renal failure may, in themselves, cause an encephalopathy. Since SAE often precedes multi-organ failure, this secondary mechanism would not explain the early encephalopathy of sepsis. However, multi-organ failure likely plays a contributory role in producing brain dysfunction in advanced sepsis.

\section{Cytokine actions in the brain}

Pro-inflammatory cytokines released by activated neutrophils and monocytes include interleukin-1 (IL-1 $\alpha$ and IL-1 $\beta$ ), IL-6 and TNF- $\alpha$. Interleukin- 1 can stimulate vagal afferents that, in turn, affect brainstem, limbic and hypothalamic structures to produce "illness behavior" characterized by decreased activity, anorexia, depressed mood and cognitive impairment. ${ }^{21}$ Interleukin- 1 stimulates the production of prostaglandin E- 2 by brain endothelial cells. ${ }^{22}$ This produces fever and activation of the hypothalamic-pituitary adrenal axis to increase cortisol production. Interleukin- $1 \beta$ may directly affect a number of brain nuclei in circumventricular organs that are not guarded by the blood-brain barrier (including the area postrema) and choroid plexus. Activation of these structures could alter limbic function in the amygdala and hypothalamus, thereby causing depression and anorexia. Administration of TNF- $\alpha$ alters the brain's metabolism of tryptophan to produce more kynurinine (that is converted to the neurotoxin quinolinic acid) and less serotonin; these biochemical changes are associated with emotional depression..$^{23}$

A recent study revealed that peripheral inflammation leads to the activation of "signal transducer and activator of transcription 3" (STAT3), first in brain cells near the blood-brain and brainCSF interfaces and then throughout much of the brain parenchyma, possibly through the mediation of proinflammatory cytokines. ${ }^{24}$. As the activated cells proved to be astrocytes, this study adds support to the concept that SAE relates to an altered interaction of astrocytes and neurons (see below).

\section{Excitotoxicity and oxidative stress}

Antibiotics often fail to prevent neurologic complications of sepsis, in part because antibiotic destruction of bacteria releases endotoxins [e.g., Escherichia coli lipopolysaccharide (LPS)] that stimulate inflammation. ${ }^{25,26}$ Lipopolysaccharide increases cerebral levels of pro-inflammatory cytokines such as interferon $\gamma$ (IFN $\gamma$ ). Together, LPS and cytokines induce the reactive phenotype in brain astrocytes and upregulate inducible nitric oxide synthase (iNOS) in these and other cell types..$^{27,28}$ Nitric oxide synthase enzymes use reduced nicotinamide adenine dinucleotide phosphate (NADPH), oxygen and arginine to produce NO and superoxide. ${ }^{29}$ These products kill invading organisms but also evoke oxidative stress in host cells. ${ }^{30}$

Septic processes can be studied in primary astrocyte cultures, where the combination of LPS and IFN $\gamma$ (i.e., LPS + IFN $\gamma$ ) induces expression of iNOS. ${ }^{31}$ Lipopolysaccharide + IFN $\gamma$ also upregulates the argininosuccinate synthetase that produces the NOS substrate L-arginine and thus enhances NO production in astrocytes. ${ }^{32}$ These changes indicate that glial argininosuccinate synthetase and iNOS contribute to the increased cerebral NO levels caused by LPS + IFN $\gamma$ in situ. ${ }^{33}$ The high NO levels inhibit 
mitochondrial respiration, at least in part by decreasing the affinity of cytochrome c oxidase for oxygen. ${ }^{34}$ Additionally, NO combines with superoxide to form peroxynitrite, which inhibits respiratory and glycolytic enzymes, leading to energy depletion in brain cells. ${ }^{35}$ Indeed, adenosine triphosphate (ATP) content drastically decreases in glial cultures exposed to LPS + IFN $\gamma{ }^{36}$ The mitochondrial dysfunction depletion may explain why sepsis-induced elevation of NO production is accompanied by increases in extracellular hydrogen peroxide. ${ }^{37}$ Lipopolysaccharide $+\mathrm{IFN} \gamma$ also increases intracellular oxidants and depletes glutathione from astrocytes. ${ }^{38}$ In mixed neuronastrocyte cultures, LPS+IFN $\gamma$ induces iNOS expression in the astrocytes and sensitizes the neurons to killing by oxygenglucose deprivation. ${ }^{39}$

Sepsis-associated encephalopathy also involves excitotoxicity by glutamate. Septicemia elevates glutamate concentration 5-fold in the brain interstitial fluid. ${ }^{40}$ Similarly, injection of LPS $^{41}$ or NO donors ${ }^{42}$ increases extracellular glutamate concentration in brain. The extracellular glutamate activates $\mathrm{N}$-methyl $\mathrm{D}$-aspartate (NMDA)-type glutamatergic receptors to extents that stimulate or injure susceptible neurons. For example, LPS elicits cardiovascular responses that can be prevented by blockade of NMDA-type glutamatergic receptors in brain. ${ }^{43}$ Furthermore, administration of an NMDA antagonist lessens the destruction of cholinergic neurons caused by LPS. ${ }^{44}$ High extracellular glutamate levels probably account for the increased incidence of seizure in critically ill patients. ${ }^{45}$ An increased concentration of extracellular glutamate also initiates a gradual down-regulation of the expression and activity of NMDA receptors. ${ }^{46}$ This impairs the responsiveness of surviving neurons to glutamate and may contribute to patients becoming obtunded.

The rise in extracellular glutamate following a septic insult may be due, at least partially, to inhibition of astrocytic clearance of this transmitter. Septic induction of iNOS is associated with inhibition of glutamate clearance in mixed neuron-astrocyte cultures, where LPS + IFN $\gamma$ potentiates the rise in extracellular glutamate concentration caused by oxygen-glucose deprivation. ${ }^{39}$ Under normal conditions, glutamate is removed from synaptic spaces by high-affinity, $\mathrm{Na}^{+}$-dependent transporters (EAAT) located on astrocytes. Inhibition of the EAAT transporters has been shown to trigger neurologic dysfunction under nonseptic conditions ${ }^{47}$ We observed that LPS + IFN $\gamma$ slows $\mathrm{Na}^{+}$-dependent glutamate uptake by astrocytes. ${ }^{31}$ When septic insults induce iNOS, the abundant peroxynitrite that is produced inhibits EAAT directly. ${ }^{48,49}$ Furthermore, the oxidants produced in immunostimulated astrocytes inhibit ATP synthesis. ${ }^{50}$ The subsequent fall in intracellular ATP concentration, by inhibiting $\mathrm{Na}^{+}, \mathrm{K}^{+}$-ATPase, may collapse the $\mathrm{Na}^{+}$gradient across the plasma membrane and thereby decrease the force driving glutamate uptake. Oxidative stress in astrocytes also leads to cell swelling and opening of channels or pores in the plasma membrane, through which glutamate exits. ${ }^{51,52}$ Thus, oxidants accelerate glutamate efflux from the cytoplasm of astrocytes, where glutamate concentration normally is 1000 -fold higher than in extracellular fluid. ${ }^{52}$

Endogenous antioxidants like ascorbate (vitamin C) prevent the injurious effects of oxidants. Ascorbate is present at millimolar concentrations in brain cells, where it reduces reactive oxygen and nitrogen species ${ }^{53}$ As it donates electrons to oxidants, ascorbate becomes oxidized to ascorbyl radical and then to dehydroascorbic acid. Subsequently, reducing equivalents derived from cell metabolism (e.g., NADPH generated by the pentose phosphate pathway of glucose metabolism) reduce the dehydroascorbic acid and ascorbyl radical to ascorbate. In sepsis, however, ascorbate may be oxidized by reactive oxygen species at rates that overwhelm the ability of cells to regenerate the vitamin. The observation that the concentration of ascorbyl radical is increased in septic patients ${ }^{54}$ is consistent with an imbalance in the redox cycling of ascorbate. Because brain cells cannot synthesize ascorbate de novo from glucose, they rely on blood and cerebrospinal fluid. This extracellular supply is diminished in sepsis because ascorbate concentrations in blood, peripheral tissues and cerebrospinal fluid fall below normal. ${ }^{55-61}$ Additionally, cellular mechanisms of ascorbate transport and redox cycling may be defective, since LPS depletes ascorbate from lung, ${ }^{58}$ adrenal glands ${ }^{62}$ and heart. ${ }^{63}$

Brain cells take up extracellular ascorbate through specific, EAAT ${ }^{64,65}$ Exposure of astrocytes to LPS + IFN $\gamma$ inhibits the initial rate of $\mathrm{Na}^{+}$-dependent ascorbate uptake. ${ }^{31} \mathrm{~A}$ faster way that astrocytes normally accumulate ascorbate is by taking up dehydroascorbic acid and reducing it to ascorbate. ${ }^{66,67}$ Some of the ascorbate is released subsequently and may become available to other brain cells. Brief (30-min) exposure to peroxyl radicals or $24 \mathrm{~h}$ exposure to LPS + IFN $\gamma$ decrease the rate of accumulation of intracellular ascorbate from extracellular dehydroascorbic acid. ${ }^{31,66}$ After dehydroascorbic acid is taken into cells it is reduced to ascorbate by cytosolic dehydroascorbic acid reductase or other enzymes, using reducing equivalents from NADPH or glutathione. ${ }^{67-69}$ It is this process of dehydroascorbic acid reduction that may be affected by septic insults. Nitric oxide synthase activity can influence dehydroascorbic acid concentration by consuming NADPH and by producing oxidants. Because NADPH is used for the reduction of glutathione disulfide to glutathione, the synthesis of NO by NOS, and the enzymatic reduction of dehydroascorbic acid to ascorbate, a scarcity of NADPH may limit dehydroascorbic acid reduction in septic cells. Furthermore, NOS activity increases the production of oxidants (e.g., peroxynitrite) that can strip electrons from ascorbate and thereby regenerate dehydroascorbic acid intracellularly. Administering a blocker of NOS activity lessens the effect of LPS + IFN $\gamma$ on dehydroascorbic acid reduction to ascorbate in astrocytes. ${ }^{31}$ Therefore, excessive production of reactive nitrogen species (e.g., peroxynitrite) during the inflammatory response to LPS + IFN $\gamma$ appears to mediate the inhibition of ascorbate accumulation observed in septic glia. Intracellular dehydroascorbic acid that is not reduced to ascorbate exits cells and thus septic astrocytes fail to clear dehydroascorbic acid from extracellular fluid. In brain, this inadequate clearance of extracellular dehydroascorbic acid may contribute to encephalopathy because high concentrations of dehydroascorbic acid are directly toxic to neurons. ${ }^{70}$

\section{Apoptosis}

Apoptosis is a form of programmed cell death that may be triggered by TNF- $\alpha$ stimulation of neuronal receptors that activate caspase enzymes. An alternative mechanism involves mitochondrial damage that triggers apoptosis. A recent study 
revealed apoptosis in central nuclei of the autonomic nervous system in patients who died having had SAE. ${ }^{20}$ Death of other cell types in various organs, e.g., endothelial cells, hepatocytes, gastrointestinal epithelial cells and cardiac myocytes) has been described, and linked to endotoxin and/or the inflammatory cytokines, especially TNF- $\alpha .^{71-73}$ It is reasonable to suppose that the CNS cells and/or the brain's capillary endothelium are affected in a similar way by TNF- $\alpha$ or another cytokine.

\section{Treatment-related causes}

We should not forget iatrogenically-induced encephalopathy. Drugs often show delayed clearance in sepsis. ${ }^{74}$ Sedative drugs, especially benzodiazepines and narcotics, may accumulate when used for several days, especially if clearance is impaired due to organ failure and endotoxin-mediated inhibition of the cytochrome P450 system. ${ }^{75}$ Some medications, e.g., penicillin in patients with renal failure, imipenim and aminophylline, may produce seizures that could be convulsive or nonconvulsive. ${ }^{76,77}$ Various fluid, electrolyte (including calcium, magnesium and phosphate) and osmotic disorders may accompany disorders of water homeostasis, enteral or parenteral feeding, renal impairment and errors in fluid replacement. ${ }^{76,78}$ Some volume expanders may produce hemorrhagic complications and hypocalcemia. ${ }^{79}$ Encephalopathies related to cardiopulmonary by-pass procedures and organ transplantations are welldocumented. ${ }^{76,80}$

\section{Clinical features}

Sepsis-associated encephalopathy is a diffuse disturbance in cerebral function. The principal neurological findings relate to altered mental status. $^{3}$ Mildly encephalopathic patients demonstrate a fluctuating confusional state and inappropriate behavior. Inattention and writing errors (including spelling, writing full sentences, orientation of writing on the page) are common. More severely affected patients show delirium, an agitated confusional state or coma.

The most common motor sign is paratonic rigidity or gegenhalten, a resistance to passive movement of limbs that is velocity-dependent: the resistance felt during movements at normal rate disappears when the limb is moved slowly. ${ }^{3}$ Asterixis, multifocal myoclonus, seizures and tremor are relatively infrequent. Cranial nerve functions are almost invariably spared. Lateralized features, such as gaze palsy or a hemiparesis are almost never encountered; such findings should prompt investigation for other etiologies, especially ones with macroscopic focal lesions.

Clinical or laboratory evidence of peripheral nerve dysfunction, namely critical illness polyneuropathy, is found in $70 \%$ of our encephalopathic patients. ${ }^{3}$ The neuropathy is axonal in type and takes many months to resolve. It is later in onset and much slower to recover than the encephalopathy. ${ }^{3}$ Clinically, one finds decreased movement and the loss of deep tendon reflexes. Motor function is usually more affected than sensation, so that the patient may grimace without other movement when a nail bed is squeezed. In severe cases, the polyneuropathy may affect the phrenic nerves, creating problems in weaning the patient from the ventilator. ${ }^{81}$

Patients with $\mathrm{SAE}$ hyperventilate (low $\mathrm{PaCO}_{2}$ ), initially related to a respiratory alkalosis and often in later stages due to

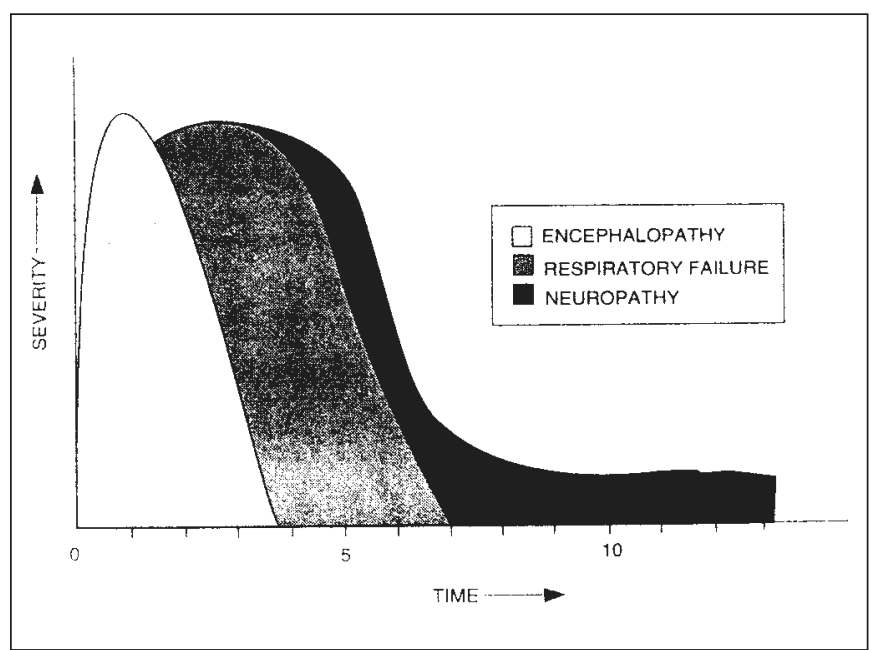

Figure 1: The typical course of nervous system complications of sepsis: encephalopathy, difficulty weaning from the ventilator, and then polyneuropathy. In the severe form this may take months, but could be weeks in milder forms. (Reproduced from Bolton CF, Young GB, Zochodne DW. The neurological complications of sepsis. Ann Neurol 1993; 33: 94-100 with permission).

a metabolic acidosis. The latter likely relates to poor tissue perfusion with increased lactate or to renal impairment as a complication of sepsis.

The relative time course of the encephalopathy, the failure to wean from the ventilator (often due to critical illness polyneuropathy or myopathy) and the resolution of critical illness polyneuropathy is illustrated in Figure 1.

\section{LABORATORY INVESTIGATIONS}

The electroencephalogram (EEG) serves as the most sensitive test for SAE. ${ }^{2}$ It may show mild, diffuse, reversible slowing in bacteremia, even if the neurological examination is normal. The severity of the EEG abnormality is parallel to the mental status impairment. As the encephalopathy worsens, mild slowing in the theta $(>4$ to $<8 \mathrm{~Hz})$ range is followed by diffuse delta $\left({ }^{2} 4 \mathrm{~Hz}\right)$ waves, then generalized triphasic waves and finally by suppression or a generalized burst-suppression (alternating diffuse reductions in voltage with burst of higher voltage waves) pattern. The relationships of EEG classifications to severity of encephalopathy and mortality are illustrated in Figures 2 and 3.

In bacteremic patients, mortality is directly related to the severity of the EEG abnormality: $0 \%$ with normal, $19 \%$ with theta, 36 with delta, $50 \%$ with triphasic waves and $67 \%$ with suppression or burst-suppression. ${ }^{2}$

Computed tomographic brain scans are normal. ${ }^{6}$ In our experience MRI scans are usually unremarkable, but occasional patients show various degrees of vasogenic edema (unpublished observations). This fits with the neuropathology and experimental studies (see above): neuronal loss and microabscesses are microscopic, but the blood-brain barrier can be disrupted in a dynamic, multifocal fashion. Cerebrospinal fluid analysis shows only a mild elevation of protein 


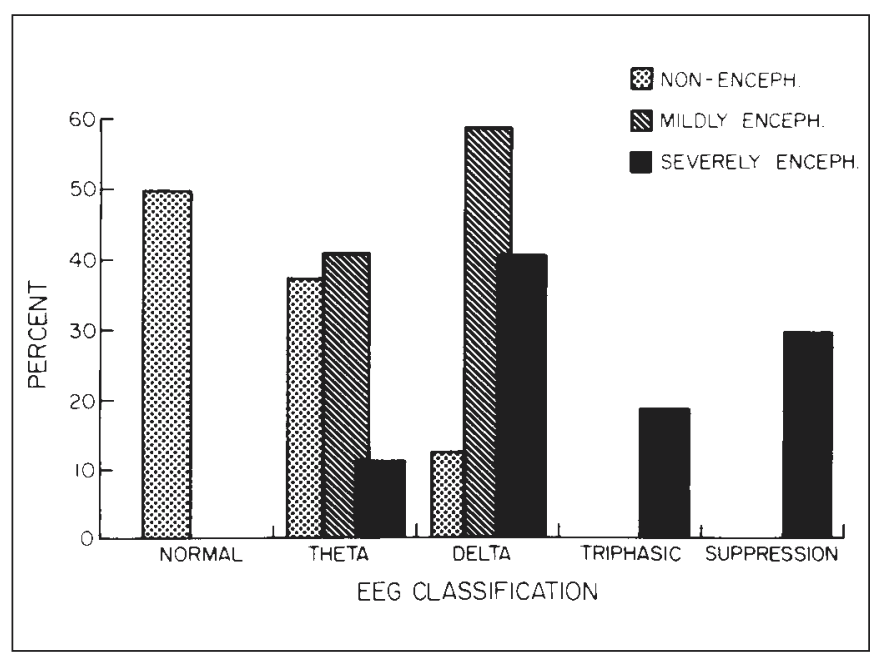

Figure 2: The histogram shows the percentages of patients with no clinical encephalopathy with bacteremia, mild septic encephalopathy, and severe septic encephalopathy with electroencephalographic (EEG) classifications: normal, theta (mild generalized slowing), delta (severe slowing), triphasic waves and generalized suppression or burstsuppression (supp). (Reproduced from Young GB, Bolton CF, Archibald $Y M$, Austin TW, Wells GA. The electroencephalogram in SAE. J Clin Neurophysiol 1992; 9: 145-152 with permission).

concentration in some, but not all, of the patients and normal cell counts and glucose concentrations, even in patients with brain microabscesses at autopsy. ${ }^{1}$

We find no significant correlation of severity of encephalopathy with any specific bacteria but there is a trend for patients with multiple bacteria on blood culture and with Candida albicans to have more severe brain dysfunction and a higher mortality rate. ${ }^{1}$

The following biochemical tests show a direct proportional

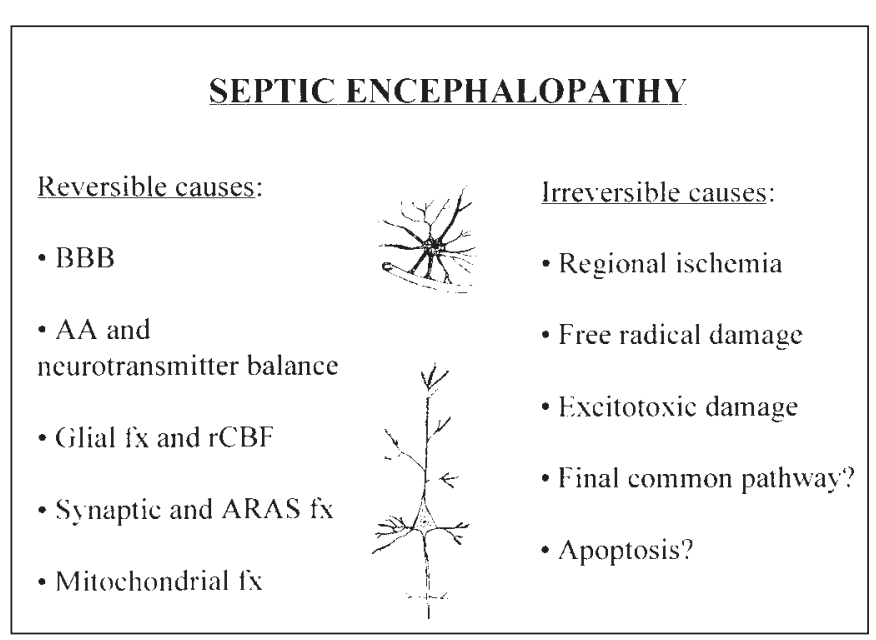

Figure 4: This cartoon represents the three principal cell types relevant to septic encephalopathy: the endothelial cell in brain capillaries, the astrocyte and the neuron. Conceptually the encephalopathy can be divided into dysfunction that is reversible or irreversible, with structural changes in the brain. The listed mechanisms are discussed in the text. $B B B=$ blood-brain barrier $; A A=$ amino acid

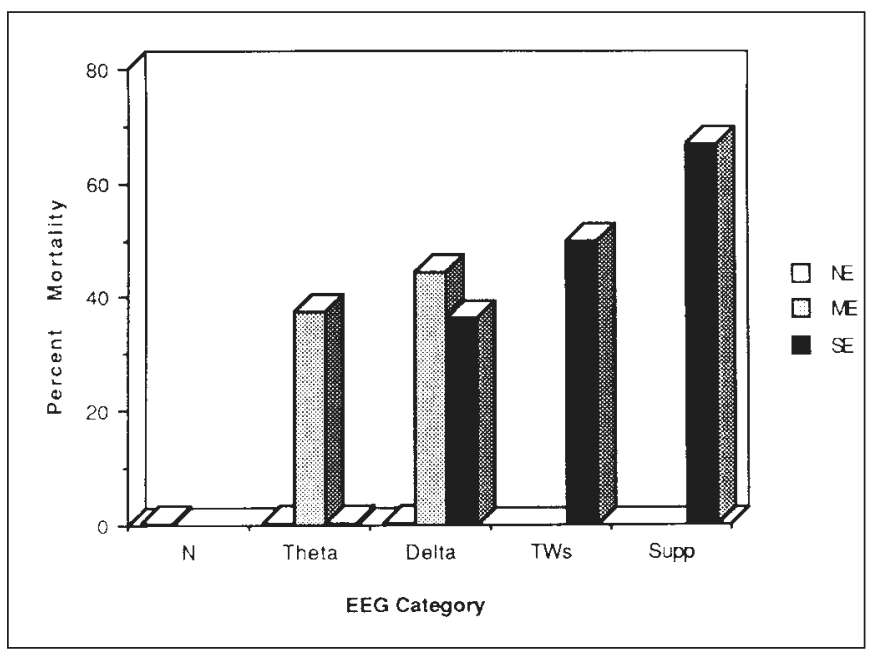

Figure 3: Histogram of mortality for the various EEG and encephalopathic states described in Figure 2. (Reproduced from Young $G B$, Bolton $C F$, Archibald YM, Austin TW, Wells GA. The electroencephalogram in SAE. J Clin Neurophysiol 1992; 9: 145-152 with permission).

$N E=$ no clinical encephalopathy with bacteremia, $M E=$ mild septic encephalopathy, $S E$ = severe emcephalopathy

change with the severity of the encephalopathy: serum urea, creatinine, bilirubin and alkaline phosphatase. The degree of renal impairment is rarely sufficient to account for the brain dysfunction. ${ }^{1}$ Hepatic failure is, however, usually difficult to assess clinically or to quantify biochemically. Encephalopathic septic patients have greater elevation of aromatic amino acids and lower concentrations of branched chain amino acids in plasma than do nonencephalopathic patients. ${ }^{15}$ This is similar to the unbalanced ratio found in hepatic failure. In sepsis, however, it may relate mainly to altered metabolism of amino acids in muscle and liver.

\section{Management}

First one must establish the diagnosis. Sepsis-associated encephalopathy is a diagnosis of exclusion: there should be no clinical or laboratory evidence of direct central nervous system infection (e.g., meningitis, macroscopic intracranial abscess or empyema), head trauma, fat embolism, adverse reactions to medications, or sedative or paralyzing drug effects. It is especially important to perform a lumbar puncture on obtunded patients with systemic inflammatory response syndrome, to rule out meningitis.

Seizures (uncommon) should be treated with standard antiepileptic medications such as phenytoin.

Patients usually die due to the severity of the systemic illness or of failure of organs other than the brain. ${ }^{1}$ Clinicians and nurses should be watchful for early encephalopathy, as it can be a presenting feature of sepsis. Prompt, specific treatment of the septic illness (appropriate antimicrobial therapy, drainage of abscesses, etc.) with prevention of multi-organ failure will save lives, as mortality is directly related to the severity of sepsis and the number of failed organs. ${ }^{82}$ With the administration of a 
mixture of amino acids with high concentrations of branchedchain amino acids, Freund and colleagues successfully reversed SAE in five patients. ${ }^{13}$ This short-term improvement is gratifying, but such treatment may not be helpful for advanced cases with multi-organ failure.

Antibiotics may fail for several reasons. First, the bacteria may be resistant. Second, the antibiotics may act too slowly, leaving sufficient time for severe inflammatory injury to develop. Third, the killing of bacteria may release inflammatory agents such as LPS.

Drugs or agents, e.g., activated protein $\mathrm{C}$, that affect or counteract the procoagulant state in sepsis may be directly or indirectly beneficial to brain function in sepsis. However, the potential benefits of this therapy on SAE directly will be difficult to separate from systemic effects.

Future therapies may combine antibiotics with drugs that slow the production of reactive nitrogen and oxygen species triggered by septic insults. For example, ascorbate is a hydrophilic antioxidant that improves survival and decreases morbidity in animals after LPS administration ${ }^{58,83}$ or peritonitis. ${ }^{61,84}$ Ascorbate also prevents LPS-induced free radical production, protein oxidation, lipid peroxidation and apoptosis in peripheral tissues. ${ }^{85-87}$ In vitro experiments have shown that adding ascorbate or ascorbate-phosphate to primary astrocyte cultures increases intracellular ascorbate concentration and attenuates iNOS induction by LPS + IFN $\gamma .^{31}$ Furthermore, intracellular ascorbate decreases inhibition of astrocytic glutamate uptake by LPS + IFN $\gamma .{ }^{31}$ Millimolar concentrations of ascorbate reduce superoxide and thereby prevent peroxynitrite formation from superoxide and $\mathrm{NO} .^{88,89}$ This effect is physiologically relevant because the steady-state intracellular ascorbate concentration can reach approximately $10 \mathrm{mM}$ in some cell types (e.g., astrocytes ${ }^{67}$ ). Ascorbate also chemically reduces peroxynitrite and other reactive oxygen and nitrogen species. ${ }^{89}$ These scavenging actions may explain how ascorbate diminishes the levels of oxidizing free radicals in LPS-exposed myocardium ${ }^{63}$ and neutrophils ${ }^{85}$ and lessens LPS-induced oxidative modification of proteins in liver ${ }^{86}$ Both ascorbate and antioxidant enzymes (superoxide dismutase and catalase) are capable of decreasing the induction by inflammatory cytokines of NOS activity in vascular endothelial cell cultures..$^{90,91}$ Pretreatment of astrocytes with ascorbate-phosphate, to elevate the intracellular concentration of ascorbate prior to LPS + IFN $\gamma$ exposure, or coadministration of an inhibitor of NOS activity, protects glutamate uptake rate. ${ }^{31}$ Taken together, these observations indicate that antioxidant effects of ascorbate may prevent iNOS induction and contribute to the preservation of normal cell function during exposure to bacterial toxins. Treatments that increase intracellular ascorbate concentration may be beneficial for patients at risk for neurologic complication in sepsis. By preserving the glutamate clearance function of astrocytes, intracellular ascorbate may allow these cells to regulate the composition of the extracellular fluid and thereby protect neurons. Systemic administration of ascorbic acid $(20 \mathrm{mg} / \mathrm{kg})$ has been shown in rats to increase the concentration of ascorbate and decrease the level of reactive oxygen species in the extracellular fluid of the striatum. ${ }^{92}$ Because intravenous injection of ascorbic acid may aggravate systemic acidosis in patients, it may be preferable to administer the sodium salt of ascorbate.
Estrogens are lipophilic antioxidants that may contribute to gender differences in sepsis, which include a significantly better prognosis for women. ${ }^{93}$ Astrocytes are essential mediators of the neuroprotective actions of estrogens after ischemia ${ }^{94}$ and may have a similar role in sepsis since, for example, 17 $\beta$-estradiol attenuates LPS-induced NF- $\mathrm{B}$ nuclear translocation in rat astrocytes. $^{95}$ The concentration of $17 \beta$-estradiol $(1 \mu \mathrm{M})$ may have been high enough to effectively scavenge free radicals and

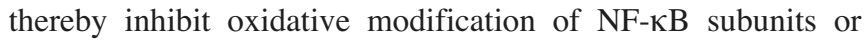
other proteins involved in cell signalling. The conjugate phenoxy radical of $17 \beta$-estradiol is unusually reactive toward ascorbate by $\mathrm{H}$-atom transfer. ${ }^{96}$ Thus estradiol and ascorbate may function together synergistically as antioxidants.

\section{Conclusion}

Sepsis-associated encephalopathy is a syndrome consisting of a diffuse disturbance in cerebral function that occurs in the context of infection in the body but without clinical or laboratory evidence of CNS infection. The mortality is highest in those with more severe cerebral dysfunction, as reflected in EEG grade. The neurological outcome is dichotomous: mild cases likely recover completely, while survivors of severe sepsis may have deficits. This reflects the varied pathology and pathogenesis of SAE. Mild cases are similar to other metabolic encephalopathies and involve reversible metabolic, neurotransmitter or microcirculatory disorders. Iatrogenic factors, often related to impaired drug clearance, play a contributory role. In severe cases, the neurons and glia may be lost due to irreversible ischemic, excitotoxic or oxidative stress or activation of apoptotic mechanisms. Although the pathogenesis remains hypothetical, recent research raises the possibility of therapeutic intervention. Eradication of the underlying infection remains the cornerstone of treatment, as this corrects the systemic inflammatory response and allows organs, including the brain, to recover. Specific strategies to spare neuronal and glial death should be considered. Those that appear promising are ones that will not compromise the general immunological defenses, but will offset oxidative stresses, focal ischemia and apoptosis in the brain. This should be testable in animal or tissue culture models and in clinical trials in humans.

\section{REFERENCES}

1. Bone RC, Sprung CL, Sibbald WJ. Definitions of sepsis and multiple organ failure. Crit Care Med 1992; 20: 724-726.

2. Young GB, Bolton CF, Austin TW, et al. The encephalopathy associated with septic illness. Clin Invest Med 1990; 13: 297304.

3. Young GB, Bolton CF, Archibald YM, Austin TW, Wells GA. The electroencephalogram in SAE. J Clin Neurophysiol 1992; 9: 145152.

4. Bolton CF, Young GB, Zochodne DW. The neurological complications of sepsis. Ann Neurol 1993; 33: 94-100.

5. Lazosky A, Young GB, Phillips R. Quality of life following severe sepsis. Crit Care Med 2002; (submitted).

6. Jackson AC, Gilbert JJ, Young GB, Bolton CF. The encephalopathy of sepsis. Can J Neurol Sci 1985; 12: 303-307.

7. Maekawa T, Fuji Y, Sadanitsu D. Cerebral circulation and metabolism in patients with septic encephalopathy. Am J Emerg Med 1981; 9: 239-245.

8. Glauser MP, Zanetti G, Baumgartner JD, Cohen J. Septic shock: pathogenesis. Lancet 1991; 732-736. 
9. Spain DA, Wilson MA, Bar-Natan MF, Garrison RN. Nitric oxide synthetase inhibition aggravates intestinal microvascular vasoconstriction and hypoperfusion of bacteremia. J Trauma 1994; 36: 720-725.

10. Vincent JL. Microvascular endothelial dysfunction: a renewed appreciation of sepsis pathophysiology. Crit Care 2001; 5: S1-S5.

11. Grinnell BW, Joyce D. Recombinant human activated protein C: a system modulator of vascular function for treatment of severe sepsis. Crit Care Med 2001; 29: S53-S60.

12. du Moulin GC, Paterson D, Hedley-White J, Broitman SA. E. coli peritonitis and bacteremia cause increased blood-brain barrier permeability. Brain Res 1995; 340: 261-268

13. Freund HR, Ryan JA, Fischer JE. Amino acid derangements in patients with sepsis: treatment with branched chain amino acid rich infusions. Ann Surg 1978; 188: 423-430.

14. Freund HR, Muggia-Sullam M, Peiser J, Melamed E. Brain neurotransmitter profile is deranged during sepsis and septic encephalopathy in the rat. J Surg Res 1985; 38: 267-271.

15. Sprung CL, Cerra FB, Freund HR, et al. Amino acid alterations and encephalopathy in the sepsis syndrome. Crit Care Med 1991; 19: 753-757.

16. Basler T, Meier-Hellmann A, Bredle D, Reinhart K. Amino acid imbalance early in septic encephalopathy. Intensive Care Med 2002; 28: 293-298

17. Schafer DF, Jones EA. Hepatic encephalopathy and the gammaaminobutyric-acid neurotransmitter system. Lancet 1982; 1: 1820.

18. Moroni F, Lombardi G, Carla V, et al. Increase in the content of quinolinic acid in cerebrospinal fluid and frontal cortex of patients with hepatic failure. J Neurochem 1986; 47: 1667-1671.

19. Mullen KD, Szauter KM, Kaminsky-Russ K. "Endogenous" benzodiazepine activity in body fluids of patients with hepatic encephalopathy. Lancet 1990; 336: 81-83.

20. Gray F, Sharshar T, De La Grandmaison GL, Annane D. Neuropathology of septic shock. Neuropathol Appl Neurobiol 2002; 28: 159.

21. Dantzer R, Konsman JP, Bluthe RM, Kelley KW. Neural and humoral pathways of communication from the immune system to the brain: parallel or convergent. Auton Neursci 2000; 85: 60-65.

22. Konsman JP, Parnet P, Dantzer R. Cytokine-induced sickness behaviour: mechanisms and implications. Trends Neurosci 2002; 25: 154-159.

23. Maes M. Major depression and the activation of the inflammatory response system. Adv Exp Med Biol 1999; 461: 25-46.

24. Gautron L, Lafon P, Chaigniau M, Tramu G, Laye S. Spatiotemporal analysis of signal transducer and activator of transcription 3 activation in rat brain astrocytes and pituitary following peripheral immune challenge. Neuroscience 2002; 112: 717-729.

25. Periti P. Current treatment of sepsis and endotoxaemia. Expert Opin Pharmacother 2000; 1: 1203-1217.

26. Lepper PM, Held TK, Schneider EM, et al. Clinical implications of antibiotic-induced endotoxin release in septic shock. Intensive Care Med 2002; 28(8): 24-33.

27. Sugaya K, Chou S, Xu SJ, McKinney M. Indicators of glial activation and brain oxidative stress after intraventricular infusion of endotoxin. Brain Res Mol Brain Res 1998; 58: 1-9.

28. Zhao ML, Liu JS, He D, Dickson DW, Lee SC. Inducible nitric oxide synthase expression is selectively induced in astrocytes isolated from the adult human brain. Brain Res 1998; 813: 402405.

29. Tolias CM, McNeil CJ, Kazlauskaite J, Hillhouse EW. Superoxide generation from constitutive nitric oxide synthase in astrocytes in vitro regulates extracellular nitric oxide availability. Free Radic Biol Med 1999; 26: 99-106.

30. Sewerynek E, Abe M, Chen L, Ortiz GG, Reiter RJ. Oxidative changes in the liver, brain and lens of lipopolysaccharide-treated rats. Arch Med Res 1995; 26: S109-S115.

31. Korcok J, Wu F, Tyml K, Hammond RR, Wilson JX. Sepsis inhibits uptake of ascorbate and redox cycling of dehydroascorbic acid: intracellular ascorbate depletion increases nitric oxide synthase induction and glutamate uptake inhibition. J Neurochem 2002; 81: 185-193.
32. Heneka MT, Schmidlin A, Wiesinger H. Induction of argininosuccinate synthetase in rat brain glial cells after striatal microinjection of immunostimulants. J Cerebral Blood Flow Metab 1999; 19: 898-907.

33. Suzuki Y, Fujii S, Numagami Y, et al. In vivo nitric oxide detection in the septic rat brain by electron paramagnetic resonance. Free Radic Res 1998; 28: 293-299.

34. Brown GC. Nitric oxide as a competitive inhibitor of oxygen consumption in the mitochondrial respiratory chain. Acta Physiol Scand 2000; 168: 667-674.

35. Bolanos JP, Almeida A, Stewart V, et alJ. Nitric oxide-mediated mitochondrial damage in the brain: mechanisms and implications for neurodegenerative diseases. J Neurochem 1997; 68: 22272240.

36. Nicoletti VG, Caruso A, Tendi EA, et al. Effect of nitric oxide synthase induction on the expression of mitochondrial respiratory chain enzyme subunits in mixed cortical and astroglial cell cultures. Biochimie 1998; 80: 871-881.

37. McNaught KS, Jenner P. Extracellular accumulation of nitric oxide, hydrogen peroxide, and glutamate in astrocytic cultures following glutathione depletion, complex I inhibition, and/or lipopolysaccharide-induced activation. Biochem Pharmacol 2000; 60: 979-988.

38. Noack H, Possel H, Rethfeldt C, Keilhoff G, Wolf G. Peroxynitrite mediated damage and lowered superoxide tolerance in primary cortical glial cultures after induction of the inducible isoform of NOS. Glia 1999; 28: 13-24.

39. Hewett SJ, Muir JK, Lobner D, Symons A, Choi DW. Potentiation of oxygen-glucose deprivation-induced neuronal death after induction of iNOS. Stroke 1996; 27: 1586-1591.

40. Guerra-Romero L, Tureen JH, Fournier MA, Makrides V, Tauber MG. Amino acids in cerebrospinal and brain interstitial fluid in experimental pneumococcal meningitis. Pediatr Res 1993; 33 : 510-513.

41. Lin HC, Wan FJ, Kang BH, Wu CC, Tseng CJ. Systemic administration of lipopolysaccharide induces release of nitric oxide and glutamate and c-fos expression in the nucleus tractus solitarii of rats. Hypertension 1999; 33: 1218-1224.

42. Lin HC, Kang BH, Wan FJ, Huang ST, Tseng CJ. Reciprocal regulation of nitric oxide and glutamate in the nucleus tractus solitarii of rats. Eur J Pharmacol 2000; 407: 83-89.

43. Yousef KA, Lang CH. Modulation of endotoxin-induced changes in hemodynamics and glucose metabolism by an N-methyl-Daspartate receptor antagonist. Shock 1994; 1: 335-342.

44. Willard LB, Hauss-Wegrzyniak B, Danysz W, Wenk GL. The cytotoxicity of chronic neuroinflammation upon basal forebrain cholinergic neurons of rats can be attenuated by glutamatergic antagonism or cyclooxygenase-2 inhibition. Exp Brain Res 2000; 134: 58-65

45. Koppel BS, Hauser WA, Politis C, van Duin D, Daras M. Seizures in the critically ill: the role of imipenem. Epilepsia 2001; 42: 1590-1593.

46. Cebers G, Cebere A, Kovacs AD, et al. Increased ambient glutamate concentration alters the expression of NMDA receptor subunits in cerebellar granule neurons. Neurochem Int 2001; 39: 151-160.

47. Lievens J, Bernal F, Forni C, Mahy N, Kerkerian-Le Goff L. Characterization of striatal lesions produced by glutamate uptake alteration: cell death, reactive gliosis, and changes in GLT1 and GADD45 mRNA expression. Glia 2000; 29: 222-232.

48. Sorg O, Horn TF, Yu N, Gruol DL, Bloom FE. Inhibition of astrocyte glutamate uptake by reactive oxygen species: role of antioxidant enzymes. Mol Med 1997; 3: 431-440.

49. Trotti D, Danbolt NC, Volterra A. Glutamate transporters are oxidant-vulnerable: a molecular link between oxidative and excitotoxic neurodegeneration? Trends Pharmacol Sci 1998; 19: 328-334.

50. Young Shin C, Woong Choi J, Ryun Ryu J, et al. Immunostimulation of rat primary astrocytes decreases intracellular ATP level. Brain Res 2001; 902: 198-204.

51. Innocenti B, Parpura V, Haydon PG. Imaging extracellular waves of glutamate during calcium signaling in cultured astrocytes. J Neurosci 2000; 20: 1800-1808. 
52. Peters CE, Korcok J, Gelb AW, Wilson JX. Anesthetic concentrations of propofol protect against oxidative stress in primary astrocyte cultures: comparison with hypothermia. Anesthesiology 2001; 94: 313-321.

53. Wilson JX. Antioxidant defense of the brain: a role for astrocytes. Can J Physiol Pharmacol 1997; 75: 1149-1163.

54. Galley HF, Davies MJ, Webster NR. Ascorbyl radical formation in patients with sepsis: effect of ascorbate loading. Free Radic Biol Med 1996; 20: 139-143.

55. Heinz-Erian P, Achmuller M, Berger $\mathrm{H}$, et al. Cerebrospinal fluid and plasma levels of vitamin C in children. Padiatr Padol 1985; 20: 49-54.

56. Galley HF, Davies MJ, Webster NR. Xanthine oxidase activity and free radical generation in patients with sepsis syndrome. Crit Care Med 1996; 24: 1649-1653.

57. Schorah CJ, Downing C, Piripitsi A, et al. Total vitamin C, ascorbic acid, and dehydroascorbic acid concentrations in plasma of critically ill patients. Am J Clin Nutr 1996; 63: 760-765.

58. Benito E, Bosch MA. Impaired phosphatidylcholine biosynthesis and ascorbic acid depletion in lung during lipopolysaccharideinduced endotoxaemia in guinea pigs. Mol Cell Biochem 1997; 175: 117-123.

59. Block F, Schwarz M. Vitamin B6 and vitamin C status in elderly patients with infections during hospitalization. Ann Nutr Metab 1997; 82: 344-352.

60. Metnitz PG, Bartens C, Fischer M, et al. Antioxidant status in patients with acute respiratory distress syndrome. Intensive Care Med 1999; 25: 180-185.

61. Armour J, Tyml K, Lidington D, Wilson JX. Ascorbate prevents microvascular dysfunction in the skeletal muscle of the septic rat. J Appl Physiol 2001; 90: 795-803.

62. Garcia R, Abarca S, Municio AM. Adrenal gland function in reversible endotoxic shock. Circ Shock 1990; 30: 365-374.

63. Rojas C, Cadenas S, Herrero A, Mendez J, Barja G. Endotoxin depletes ascorbate in the guinea pig heart. Protective effects of vitamins $\mathrm{C}$ and E against oxidative stress. Life Sci 1996; 59: 649657.

64. Castro M, Caprile T, Astuya A, et al. High-affinity sodium-vitamin C co-transporters (SVCT) expression in embryonic mouse neurons. J Neurochem 2001; 78: 815-823.

65. Korcok J, Yan R, Siushansian R, Dixon SJ, Wilson JX. Sodiumascorbate cotransport controls intracellular ascorbate concentration in primary astrocyte cultures expressing the SVCT2 transporter. Brain Res 2000; 881: 144-151.

66. Daskalopoulos R, Korcok J, Tao L, Wilson JX. Accumulation of intracellular ascorbate from dehydroascorbic acid by astrocytes is decreased after oxidative stress and restored by propofol. Glia 2002; 39: 124-132.

67. Siushansian R, Tao L, Dixon SJ, Wilson JX. Cerebral astrocytes transport ascorbic acid and dehydroascorbic acid through distinct mechanisms regulated by cyclic AMP. J Neurochem 1997; 68: 2378-2385

68. Rose RC. Cerebral metabolism of oxidized ascorbate. Brain Res 1993; 628: 49-55.

69. Fornai F, Saviozzi M, Piaggi S, et al. Localization of a glutathionedependent dehydroascorbate reductase within the central nervous system of the rat. Neuroscience 1999; 94: 937-948.

70. Song JH, Shin SH, Ross GM. Oxidative stress induced by ascorbate causes neuronal damage in an in vitro system. Brain Res 2001; 895: 66-72.

71. Moazzam FN, Bremm JJ, Yong SL, et al. Endotoxin potentiates hepatocyte apoptosis in cholestasis. J Am Coll Surg 2002; 194: 731-739.

72. Hotchkiss RS, Tinsley KW, Swanson PE, Karl IE. Endothelial cell apoptosis in sepsis. Crit Care Med 2002; 30: S225-S228.

73. Chen G, Goeddel DV. TNF-R1 signaling: a beautiful pathway. Science 2002; 296: 1634-1635.

74. Mandell GL, Sandle MA. Antimicrobial agents: penicillins, cephalosporins and other beta-lactam antibiotics. In: Gilman AG,
Goodman LS, Rall TW, Murad F, (Eds). Goodman and Gilman's The Pharmacological Basis of Therapeutics. 7th ed. New York: Macmillan, 1985: 1115-1149.

75. Shedlofsky SI, Israel BC, McClain CJ, et al. Endotoxin administration to humans inhibits cytochrome P450-mediated drug metabolism. J Clin Invest 1994; 94: 2209-2214.

76. Wijdicks EFM, Sharbrough FW. New-onset seizures in critically ill patients. Neurology 1993; 43: 1024-1042.

77. Pauloucek FP, Rodvold KA. Evaluation of theophylline overdoses and toxicities. Ann Emerg Med 1988; 17: 135-144.

78. Wesiinger JR, Bellorin-Font E. Magnesium and phosphorous. Lancet 1988; 352: 391-396.

79. Haynes GR, Bailey MK. Excessive use of hetastarch: an iatrogenic cause of bleeding and hypocalcemia. Anesth Analg 2000; 90 : $1455-1456$

80. Slogoff S, Giris KZ, Keats AS. Etiologic factors in neuropsychologic complications associated with cardiopulmonary bypass. Anesth Analg 1982; 61: 903-911.

81. Zochodne DW, Bolton CF, Wells GA, et al. Critical illness polyneuropathy: a complication of sepsis and multiple organ failure. Brain 1987; 110: 819-842.

82. Pine RW, Wertz MJ, Lennard ES. Determinants of organ malfunction or death in patients with intra-abdominal sepsis. Arch Surg 1983; 118: 242-249.

83. Nonaka A, Manabe T, Tobe T. Effect of a new synthetic free radical scavenger, 2-octadecyl ascorbic acid, on the mortality in mouse endotoxemia. Life Sci 1990; 47: 1933-1939.

84. Armour J, Tyml K, Lidington D, Wilson JX. Ascorbate protects the microvasculature in septic skeletal muscle. FASEB J 2001; 15: A50

85. Dwenger A, Funck M, Lueken B, Schweitzer G, Lehmann U. Effect of ascorbic acid on neutrophil functions and hypoxanthine/ xanthine oxidase-generated, oxygen-derived radicals. Eur J Clin Chem Clin Biochem 1992; 30: 187-191.

86. Cadenas S, Rojas C, Barja G. Endotoxin increases oxidative injury to proteins in guinea pig liver: protection by dietary vitamin $\mathrm{C}$. Pharmacol Toxicol 1998; 82: 11-18.

87. Bowie AG, O'Neill LA. Vitamin C inhibits NF-кB activation by TNF via the activation of $\mathrm{p} 38$ mitogen-activated protein kinase. $\mathrm{J}$ Immunol 2000; 165: 7180-7188.

88. Jackson TS, Xu AM, Vita JA, Keaney JF. Ascorbate prevents the interaction of superoxide and nitric oxide only at very high physiological concentrations. Circ Res 1998; 83: 916-922.

89. Kirsch $\mathbf{M}$, de Groot $\mathrm{H}$. Ascorbate is a potent antioxidant against peroxynitrite-induced oxidation reactions - Evidence that ascorbate acts by re-reducing substrate radicals produced by peroxynitrite. J Biol Chem 2000; 275: 16702-16708.

90. Galley HF, Walker BE, Howdle PD, Webster NR. Regulation of nitric oxide synthase activity in cultured human endothelial cells: effect of antioxidants. Free Radic Biol Med 1996; 21: 97-101.

91. Wu F, Wilson JX, Tyml K. Ascorbate inhibits iNOS expression in endotoxin- and IFN $\gamma$-stimulated skeletal muscle endothelial cells. FEBS Lett 2002; 520: 122-126.

92. Huang M, Liu W, Li Q, Wu CF. Endogenous released ascorbic acid suppresses ethanol-induced hydroxyl radical production in rat striatum. Brain Res 2002; 944: 90-96.

93. Schroder J, Kahlke V, Staubach KH, Zabel P, Stuber F. Gender differences in human sepsis. Arch Surg 1998; 133: 1200-1205.

94. Azcoitia I, Garcia-Ovejero D, Chowen JA, Garcia-Segura LM. Astroglia play a key role in the neuroprotective actions of estrogen. Prog Brain Res 2001; 132: 469-478.

95. Dodel RC, Du Y, Bales KR, Gao F, Paul SM. Sodium salicylate and

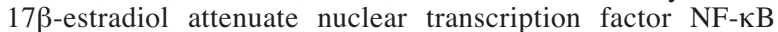
translocation in cultured rat astroglial cultures following exposure to amyloid A $\beta(1-40)$ and lipopolysaccharides. J Neurochem 1999; 73: 1453-1460.

96. Winterle JS, Mill T, Harris T, Goldbeck RA. Absolute kinetic characterization of $17 \beta$-estradiol as a radical-scavenging, antioxidant synergist. Arch Biochem Biophys 2001; 392: 233-244. 